(1)

CrossMark

\title{
Acute air pollution exposure alters neutrophils in never-smokers and at-risk humans
}

\author{
Denise J. Wooding ${ }^{1}$, Min Hyung Ryu ${ }^{1}$, Hang $\mathrm{Li}^{1,2}$, Neil E. Alexis ${ }^{3}$, Olga Pena ${ }^{1}$ and \\ Chris Carlsten ${ }^{1}$, Canadian Respiratory Research Network
}

\begin{abstract}
Affiliations: ${ }^{1}$ Air Pollution Exposure Laboratory, Dept of Medicine, Division of Respiratory Medicine, The University of British Columbia, Vancouver, BC, Canada. ${ }^{2}$ Dept of Otolaryngology, The First Affiliated Hospital of Sun Yat-sen University, Guangzhou, P.R. China. ${ }^{3}$ UNC Center for Environmental Medicine Asthma and Lung Biology, UNC Chapel Hill, Chapel Hill, NC, USA.
\end{abstract}

Correspondence: Chris Carlsten, 2775 Laurel St, 7th Floor, the Lung Center, Vancouver General Hospital Gordon and Leslie Diamond Health Care Centre, Vancouver, BC V5Z 1M9, Canada. E-mail: carlstendamail.ubc.ca

@ERSpublications

Acute human exposure to traffic-related air pollution leads lower airway neutrophils to release neutrophil extracellular traps, linked to COPD severity and airflow limitation. Ex-smokers and COPD patients mount a greater neutrophil response to exposure. http://bit.ly/2DEsYzE

Cite this article as: Wooding DJ, Ryu $\mathrm{MH}, \mathrm{Li} \mathrm{H}$, et al. Acute air pollution exposure alters neutrophils in never-smokers and at-risk humans. Eur Respir J 2020; 55: 1901495 [https://doi.org/10.1183/ 13993003.01495-2019].

ABSTRACT Outdoor air pollution exposure increases chronic obstructive pulmonary disease (COPD) hospitalisations, and may contribute to COPD development. The mechanisms of harm, and the extent to which at-risk populations are more susceptible are not fully understood. Neutrophils are recruited to the lung following diesel exhaust exposure, a model of traffic-related air pollution (TRAP), but their functional role in this response is unknown. The purpose of this controlled human-exposure crossover study was to assess the effects of acute diesel exhaust exposure on neutrophil function in never-smokers and at-risk populations, with support from additional in vitro studies.

18 participants, including never-smokers $(n=7)$, ex-smokers $(n=4)$ and mild-moderate COPD patients $(\mathrm{n}=7)$, were exposed to diesel exhaust and filtered air for $2 \mathrm{~h}$ on separate occasions, and neutrophil function in blood $(0 \mathrm{~h}$ and $24 \mathrm{~h}$ post-exposure) and bronchoalveolar lavage ( $24 \mathrm{~h}$ post-exposure) was assessed.

Compared to filtered air, diesel exhaust exposure reduced the proportion of circulating band cells at $0 \mathrm{~h}$, which was exaggerated in COPD patients. Diesel exhaust exposure increased the amount of neutrophil extracellular traps (NETs) in the lung across participants. COPD patients had increased peripheral neutrophil activation following diesel exhaust exposure. In vitro, suspended diesel exhaust particles increased the amount of NETs measured in isolated neutrophils. We propose NET formation as a possible mechanism through which TRAP exposure affects airway pathophysiology. In addition, COPD patients may be more prone to an activated inflammatory state following exposure.

This is the first controlled human TRAP exposure study directly comparing at-risk phenotypes (COPD and ex-smokers) with lower-risk (never-smokers) participants, elucidating the human susceptibility spectrum.

\section{This article has supplementary material available from erj.ersjournals.com}

This study is registered at www.clinicaltrials.gov with identifier NCT02236039. Individual participant data will not be made available.

Received: 30 July 2019 | Accepted after revision: 26 Nov 2019

Copyright CERS 2020 


\section{Introduction}

Outdoor air pollution is a significant contributor to global morbidity and is responsible for 3.3 million premature deaths yearly worldwide [1,2]. Traffic-related air pollution (TRAP) is one of the most significant sources of outdoor air pollution exposure in developed countries $[1,2]$. Chronic obstructive pulmonary disease (COPD) is the third leading cause of global years of life lost to non-communicable disease [3]. Epidemiological studies consistently demonstrate a strong association between airborne pollutant levels and COPD exacerbations requiring hospitalisation [4-8], indicating that COPD patients may be more susceptible to the harmful effects of pollutant exposure than the general population. In addition, populations susceptible to the harmful effects of air pollution exposure may extend beyond those living with disease, including ex-smokers without COPD, as well as children and adolescents [9].

The mechanisms through which air pollution exerts it harmful effects, and how these may differ in susceptible populations, are not completely understood. While a number of controlled human exposure studies to diesel exhaust, a model of TRAP, described inflammation and oxidative stress as key mechanisms [10-12], no studies of this kind have ever included at-risk groups such as COPD patients and ex-smokers to delineate the underlying mechanisms of their potential susceptibility to these exposures.

Neutrophils are recruited to the lung following acute controlled human exposure to diesel exhaust [12-15]. However, very little is known about their functional role in this response. Neutrophils play a key role in protection through their antimicrobial defences [16], but a high level of neutrophil activation is a hallmark of COPD correlating with disease severity [17]. Neutrophil extracellular traps (NETs) consist of neutrophil-derived DNA that is released from neutrophils in chromatin filaments forming web-like structures coated with granular histone protein [18]. NETs mediate a wide range of inflammatory diseases, including asthma [19] and COPD, where they are found in high concentrations in the airways and correlate with airflow limitation [20-22].

The primary purpose of this controlled human-exposure crossover study was to determine the effect of acute TRAP (diesel exhaust) exposure on neutrophil function in the blood and lower airways in humans. Second, we aimed to assess whether at-risk populations, namely ex-smokers and COPD patients, experienced greater effects of diesel exhaust exposure relative to never-smokers. We hypothesised that diesel exhaust exposure increases neutrophil activation and NET formation and decreases peripheral band cell levels, and these effects are enhanced in ex-smokers and those with COPD.

\section{Methods}

Human exposure study design

In this randomised double-blind controlled human exposure crossover study, 18 participants were exposed for $2 \mathrm{~h}$ on separate occasions to diluted diesel exhaust and filtered air (figure 1). Exposures were randomised to order by an automated algorithm, with a minimum 4-week washout period, and took place at the Air Pollution Exposure Laboratory at Vancouver General Hospital (Vancouver, BC, Canada),

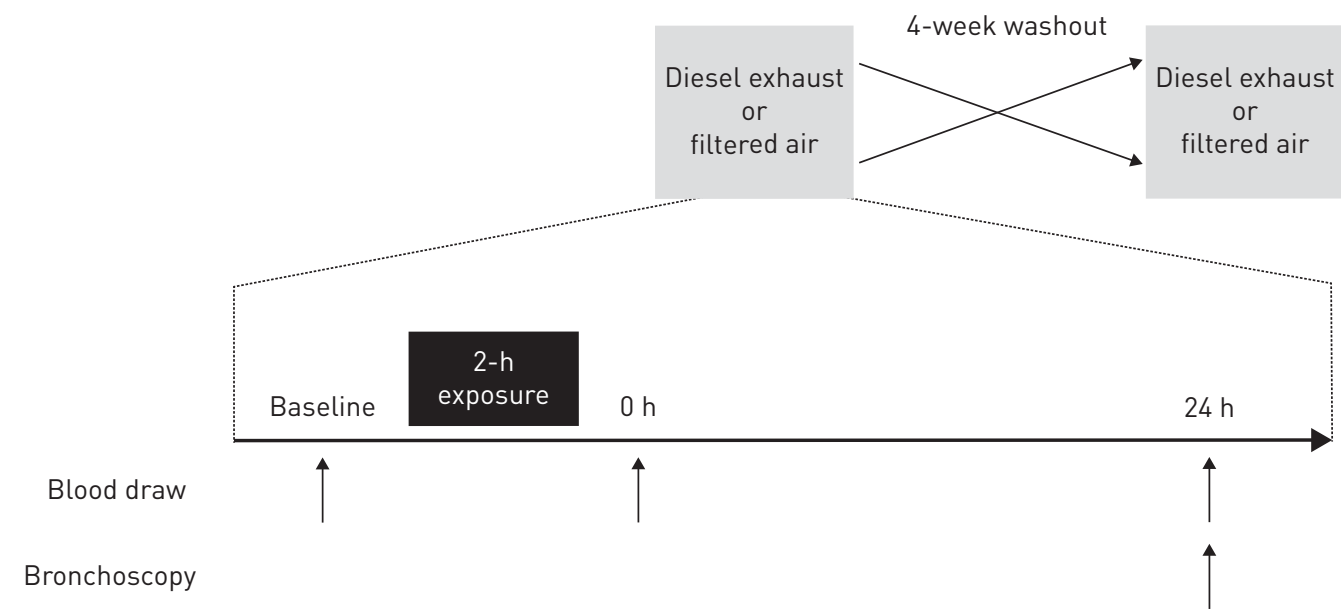

FIGURE 1 Study design. This was a randomised controlled human exposure crossover study to diesel exhaust ( $300 \mu \mathrm{g} \cdot \mathrm{m}^{-3}$ particles with a $50 \%$ cut-off aerodynamic diameter of $2.5 \mu \mathrm{m}$ ) and filtered air. 18 participants from three phenotypes ( $n=7$ never-smokers, $n=5$ ex-smokers without chronic obstructive pulmonary disease (COPD), $\mathrm{n}=6$ mild-moderate COPD) were exposed for $2 \mathrm{~h}$ on separate occasions to diesel exhaust and filtered air, in random order, separated by $\geqslant 4$ weeks. Blood samples were obtained before and after exposure, and bronchoalveolar lavage was obtained during a bronchoscopy procedure $24 \mathrm{~h}$ after exposure. 
between August 2017 and May 2019. Participants and investigators were blind to the exposure. Participants abstained from any short- or long-acting bronchodilator use, inhaled corticosteroid use and nutritional supplements for $48 \mathrm{~h}$, and caffeine for $12 \mathrm{~h}$ prior to exposures, and visits were rescheduled if participants exhibited signs of upper respiratory tract infection (Common Cold Questionnaire). Sample size was determined from previous controlled human exposure study data demonstrating a change in blood neutrophil counts after diesel exhaust exposure with 15 participants [13]. Additional details for the methods described herein, including detailed exclusion criteria, antibody details, flow cytometry controls and methods for quality control in cell counting, are available in the supplementary material.

\section{Participants}

Participants aged 40-80 years were recruited via local online classified advertisements, flyers, clinical referrals and newspaper advertisements. An initial telephone screening identified eligible participants from three phenotypes: never-smokers, ex-smokers without COPD (minimum 10 pack-years smoking history and quit $\geqslant 6$ months prior), and mild-moderate COPD patients (quit smoking $\geqslant 6$ months prior). Individuals then visited the lab for an in-person screening visit to assess baseline spirometry, confirm participant phenotype (COPD if forced expiratory volume in $1 \mathrm{~s}\left(\mathrm{FEV}_{1}\right) /$ forced vital capacity (FVC) $<0.7$ and $\mathrm{FEV}_{1} \geqslant 50 \%$ predicted) and study enrolment if eligible (table 1). All participants self-reported $\geqslant 6$ months of smoking cessation prior to study enrolment, which was continued throughout the study. Serum cotinine was measured at each exposure visit to verify abstinence from smoking, which remained $<10 \mathrm{ng} \cdot \mathrm{mL}^{-1}$ for all participants (Cotinine ELISA Kit; OriGene Technologies, Rockville, MD, USA).

\section{Exposures}

Exposures were $2 \mathrm{~h}$ in duration and followed a previously described protocol with endotoxin levels below a limit of detection of 0.52 endotoxin units $\mathrm{m}^{-3}$ (table 2) [23]. Diesel exhaust is a robust model of TRAP, and a dose approximating $300 \mu \mathrm{g} \cdot \mathrm{m}^{-3}$ of particulate matter sized $\leqslant 2.5 \mu \mathrm{m}$ was used to represent exposure levels documented in polluted mega-cities and occupational exposures [23, 24]. During exposures, participants cycled on a stationary bike for two bouts of $15 \mathrm{~min}$ at $30 \%$ maximal oxygen uptake to simulate a breathing pattern of typical outdoor daily activity such as walking.

\section{Sampling}

Blood samples were collected into EDTA tubes, placed on ice then processed immediately. A bronchoscopy was performed at $24 \mathrm{~h}$, and bronchoalveolar lavage (BAL) from the upper lobe was obtained with $2 \times 50 \mathrm{~mL}$ or $2 \times 60 \mathrm{~mL}$ instillations of saline, obtained from the opposite lobe with consistent volume in each of the two exposures. Samples were kept on ice and processed immediately. BAL was spun and cells were resuspended in RPMI medium for assay.

\begin{tabular}{|c|c|c|c|c|}
\hline & Sex & Age years & Smoking history pack-years & Post-bronchodilator $\mathrm{FEV}_{1} / \mathrm{FVC}$ \\
\hline Never-smoker & M & 63 & & 78 \\
\hline Never-smoker & M & 58 & & 75 \\
\hline Never-smoker & $\mathrm{F}$ & 48 & & 81 \\
\hline Never-smoker & $\mathrm{F}$ & 72 & & 80 \\
\hline Never-smoker & M & 50 & & 79 \\
\hline Never-smoker & $\mathrm{F}$ & 42 & & 86 \\
\hline Never-smoker & $\mathrm{F}$ & 56 & & 81 \\
\hline Ex-smoker & M & 73 & 30 & 91 \\
\hline Ex-smoker & M & 70 & 90 & 72 \\
\hline Ex-smoker & M & 63 & 47 & 70 \\
\hline Ex-smoker & M & 56 & 50 & 74 \\
\hline COPD & M & 80 & 10 & 54 \\
\hline COPD & $\mathrm{F}$ & 66 & 24 & 54 \\
\hline COPD & $\mathrm{F}$ & 70 & 12 & 48 \\
\hline COPD & $M$ & 67 & 52.5 & 49 \\
\hline COPD & $M$ & 65 & 45 & 41 \\
\hline COPD & $M$ & 75 & 7 & 67 \\
\hline COPD & $M$ & 70 & 19.5 & 64 \\
\hline
\end{tabular}

$\mathrm{FEV}_{1}$ : forced expiratory volume in $1 \mathrm{~s}$; FVC: forced vital capacity; COPD: chronic obstructive pulmonary disease; M: male; F: female. 


\begin{tabular}{|c|c|c|c|c|c|c|c|}
\hline Filtered air & $4 \pm 3$ & $211 \pm 173$ & $20 \pm 16$ & $230 \pm 184$ & $2 \pm 1$ & $2147 \pm 2476$ & $158 \pm 110$ \\
\hline
\end{tabular}

\section{Peripheral blood and BAL neutrophil flow cytometry}

Blood and BAL were stained for CD45, CD16, CD66b, activated CD11b (Mac-1) and CXCR2. In blood and BAL [25], live neutrophils were identified as $\mathrm{CD}^{+} 5^{+}, \mathrm{CD}^{+} 6^{+}, \mathrm{CD}_{6} 6 \mathrm{~b}^{+}$, LIVE/DEAD ${ }^{-}$events, which were assessed for their expression of CD16, CD66b and CXCR2, quantified as the change from baseline median fluorescence intensity (MFI) across the exposure. The percentage of neutrophils expressing an activated form of CD11b (Mac-1; CBRM 1/5 clone), which may by expressed only after a signal is received from a stimulus such as a chemoattractant, was also quantified.

\section{Peripheral blood neutrophil and band cell differential}

500 leukocytes per time point were identified in blood smears by a skilled observer (quality control is detailed in the supplementary material) as mature polymorphonuclear cells, band cells, eosinophils or others.

\section{BAL neutrophil extracellular traps}

150000 BAL cells were seeded onto uncoated round coverglass, fixed, blocked and stained for the hallmark NET markers H3 citrullinated histones (H3cit), neutrophil elastase and Hoechst 33342 DNA stain. A $4 \times 4$ grid of photos for each slide was acquired at $\times 60$ magnification and stitched into one large image (EVOS FL Auto Imaging System; Thermo Fisher Scientific, Waltham, MA, USA). NETs were defined as structures meeting the following three criteria: 1) clear extracellular fibrous citrullinated histone strands; 2) positive DNA stain; and 3) positive neutrophil elastase stain. NETs were quantified as NET-covered area (by tracing) normalised to total nuclear area (by automatic quantification on binary image) and expressed as a percentage of total nuclear area.

\section{In vitro study design}

Neutrophils were isolated from peripheral blood of four consenting male volunteers ( $\mathrm{n}=2$ healthy never-smokers, $\mathrm{n}=2$ patients with mild asthma) using magnetic negative selection (EasySep direct human neutrophil isolation kit; StemCell Technologies, Vancouver, BC, Canada) then resuspended in RPMI medium supplemented with $2 \%$ fetal bovine serum. Cells were stimulated in vitro with diesel exhaust particles (DEPs) obtained from the diesel engine exhaust outlet of the Air Pollution Exposure Laboratory after suspension in the same media and sonication to prevent agglomeration.

\section{Peripheral blood neutrophil extracellular traps by flow cytometry and immunocytochemistry}

For flow cytometric quantification of NETs stimulated in vitro, $1 \times 10^{6}$ isolated neutrophils were stimulated for $2 \mathrm{~h}$ in vitro with $100 \mu \mathrm{g} \cdot \mathrm{mL}^{-1}$ DEPs, $4 \mu \mathrm{M}$ ionomycin (positive control) or medium alone (negative control). Using a previously described flow cytometry assay [26], NETs were quantified as the percentage of cells staining triple-positive, without a permeabilisation step, for myeloperoxidase (MPO), H3Cit and DAPI DNA stain (all antibody details are listed in the supplementary material). To visualise NETs by immunocytochemistry, 120000 isolated neutrophils were seeded for $1 \mathrm{~h}$ in an incubator onto uncoated, round coverglass, then stimulated for $2 \mathrm{~h}$ with $100 \mu \mathrm{g} \cdot \mathrm{mL}^{-1} \mathrm{DEPs}, 4 \mu \mathrm{M}$ ionomycin, DEPs+ionomycin at these same concentrations or medium. Cells were fixed, blocked and stained in the same manner as the BAL NET assay, except neutrophil elastase was used at the protein marker instead of MPO. NETs were assessed qualitatively using confocal microscopy (Zeiss LSM780 laser scanning confocal microscope; Oberkochen, Germany).

\section{Statistics}

Human exposure study data were analysed using mixed effects models (nlme package, version 3.1-131) in RStudio (version 1.1.383; https://rstudio.com). The primary outcome was to assess the fixed effect of exposure (diesel exhaust versus filtered air) on each end-point, with participant ID as the random effect. The secondary outcome was to assess whether participant phenotype (never-smoker, ex-smoker, COPD) modified the effect of exposure (exposure $\times$ phenotype interaction), with participant ID as the random 
effect. The potential for order effect was assessed, with order (filtered air first versus diesel exhaust first) as the fixed effect and participant ID as the random effect. p-values $<0.05$ were considered statistically significant. In vitro data and baseline neutrophil counts were analysed in Prism 6 (GraphPad Software, La Jolla, CA, USA) using a repeated-measures one-way ANOVA and Sidak's multiple comparisons test.

\section{Study approval}

This study was approved by the University of British Columbia clinical research ethics board (H14-00821) and Vancouver Coastal Health Research Institute (V14-00821). All participants provided written informed consent prior to inclusion.

\section{Results \\ Peripheral blood neutrophil surface marker activation was greater in COPD after diesel exhaust exposure}

Cell surface expression was quantified as MFI and data are presented as mean effect (95\% CI; p-value) for interaction effect, where MFI values represent $\Delta$ (post-exposure minus pre-exposure for diesel exhaust minus filtered air). Patient phenotype significantly modified the effect of diesel exhaust exposure in blood, such that individuals with COPD had increased peripheral neutrophil CD16 (MFI +262, 113-414; $\mathrm{p}=0.006)$ and CXCR2 expression (MFI $+292,150-434 ; \mathrm{p}=0.002)$, and increased percentage of activated CD11b-expressing neutrophils $(+12.2 \%,-152-22 ; \mathrm{p}=0.046)$ relative to never-smokers $24 \mathrm{~h}$ after diesel exhaust exposure (figure 2). There was no main effect of diesel exhaust exposure and no modifying effect of order on activation marker expression in peripheral blood or BAL neutrophils, and patient phenotype did not significantly modify the effect of diesel exhaust on neutrophil surface markers in the BAL.

\section{Peripheral blood band cell and neutrophil counts decreased after diesel exhaust exposure}

Data are presented as mean values (\%) and 95\% CI where percentages represent the change in $\Delta$ (diesel exhaust (post- minus pre-exposure) versus filtered air (post- minus pre-exposure)). Circulating band cells decreased immediately following diesel exhaust exposure, when expressed as a percentage of total neutrophils (mature+band) $(-2.1 \%,-0.3--4.0 \%$; $=0.04)$ (figure 3). At $24 \mathrm{~h}$, diesel exhaust decreased the percentage of total neutrophils $(-6.2 \%,-0.82--11.6 \%$; $\mathrm{p}=0.04)$, but not band cells. Furthermore, the reduction in peripheral band cells immediately following exposure was modified by patient phenotype, showing a greater reduction in band cell percentage in COPD patients relative to never-smokers, when measured as a percentage of total cells $(-2.8 \%,-5.0--0.42 \%$; $\mathrm{p}=0.04)$. There was no effect modification by order.

\section{Diesel exhaust exposure increased the amount of NETs detectable in the lower airways}

Diesel exhaust exposure increased the amount of NETs in BAL (figure 4), when measured as the proportion (\%) of DNA-covered area that was associated with markers of NETs $(+7.5 \%, 0.6-14.3 \%$; $\mathrm{p}=0.04$ ). There was no modifying effect by patient phenotype or order.

\section{Baseline inflammatory state did not predict high and low responders}

For outcomes where the effect of diesel exhaust was modified by patient phenotype (peripheral blood band cell counts and peripheral blood CXCR2 expression), or where there was a clear high-responding group (BAL NETs), we sought to assess whether this was attributable to an elevated baseline inflammatory state (defined by baseline peripheral blood neutrophil counts). However, there was no significant difference between the three patient phenotypes in baseline neutrophil counts (supplementary figure S1), and baseline blood neutrophil counts did not modify the effect of diesel exhaust on the outcomes described above when assessed as an interaction term in our mixed effects model.

\section{DEPs induce NET formation in vitro}

Given inherent challenges in accurately assessing NETs [27], multiple methods were used to assess the presence of NETs after in vitro stimulation with DEPs. For quantitative analyses, flow cytometry was employed [26], permitting rapid and robust quantification of in vitro samples independent of observer bias. In addition, qualitative visual assessment of NET-like morphology and the presence of NET markers was confirmed using immunocytochemistry. There was a significant increase in the percentage of neutrophils forming NETs measured by flow cytometry after in vitro stimulation of peripheral neutrophils with DEPs $(37 \%, 19-55 \%$; $\mathrm{p}<0.01)$, ionomycin $(26 \%, 13-39 \%$; $<<0.05)$ and the combination of DEPs and ionomycin (51\%, 20-83\%; $\mathrm{p}<0.001$ ) (figure 5). Combined stimulation with DEPs and ionomycin augmented NET formation relative to ionomycin alone $(26 \%, 2-50 \%$; $\mathrm{p}<0.05)$. NET-like structures from these samples could also be visualised in immunocytochemistry images. 

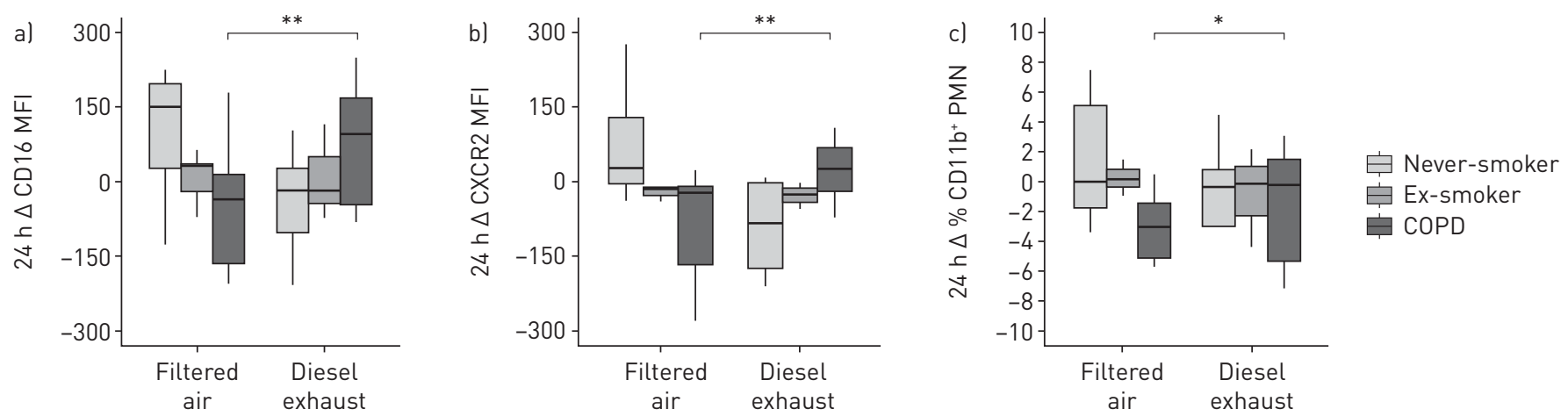

FIGURE 2 Patient phenotype modifies peripheral neutrophil activation $24 \mathrm{~h}$ after diesel exhaust exposure. The effect of acute diesel exhaust exposure on peripheral neutrophil a) CD16 and b) CXCR2 expression, and c) the percentage of polymorphonuclear cells (PMNs) expressing the activated form of CD11b (n=17) was assessed using a linear mixed effects model with patient phenotype as an interaction term and participant ID as random effect. Neutrophil activation was significantly modified by patient phenotype, such that those with chronic obstructive pulmonary disease (COPD) demonstrated increased expression in CD16, CXCR2 and CD11b expression relative to the change in never-smokers. a, b) y-axis denotes the change in expression from previous-day baseline sample $(\Delta$ : post-exposure minus pre-exposure), measured as median fluorescence intensity (MFI), and normalised to an unstained sample by subtraction. ${ }^{*}: p<0.05,{ }^{* *}$ : $p<0.01$.

\section{Discussion}

This is the first controlled human TRAP exposure study to examine at-risk COPD and ex-smoker phenotypes, along with healthy never-smokers, allowing for a direct comparison of exposure responses across the human susceptibility spectrum. Additionally, this study examined the functional role of

FIGURE 3 Diesel exhaust reduces the percentage of peripheral blood band cells and neutrophils. al Differential neutrophil and band cell counts from blood smears $(\mathrm{n}=17)$ taken before exposure, at $0 \mathrm{~h}$ (immediately after exposure) and $24 \mathrm{~h}$ for all participants. Acute human exposure to diesel exhaust reduced the percentage of circulating band cells at $0 \mathrm{~h}$, and decreased the percentage of circulating total neutrophils (band +mature) $24 \mathrm{~h}$ after exposure, compared to filtered air. b) The effect of exposure at $0 \mathrm{~h}$ was modified by patient phenotype, such that the decrease in band cells was greater in those with chronic obstructive pulmonary disease (COPD) than never-smokers. Effects were analysed using a linear mixed effects model. Values are expressed as the change from same-exposure baseline ( $\Delta$ =post-exposure minus pre-exposure), circles represent individuals. *: $p<0.05$ a)
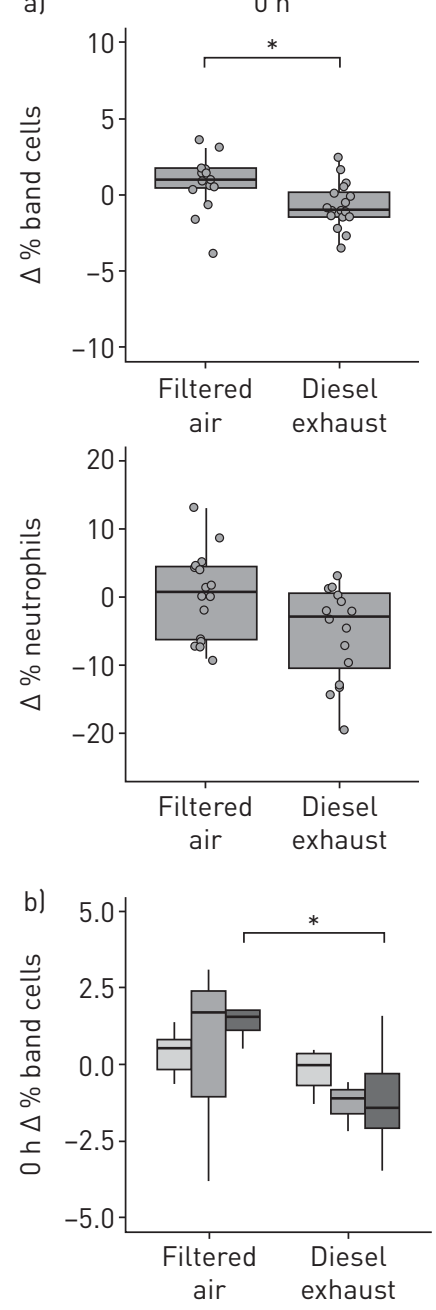

$24 \mathrm{~h}$

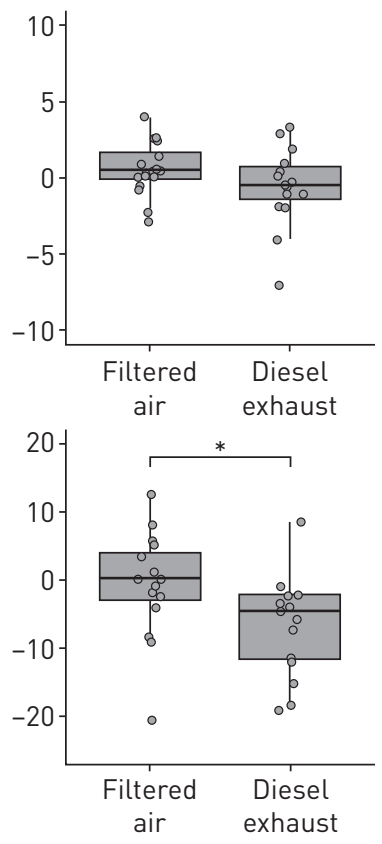

ĐNever-smoker

Ex-smoker

由COPD 

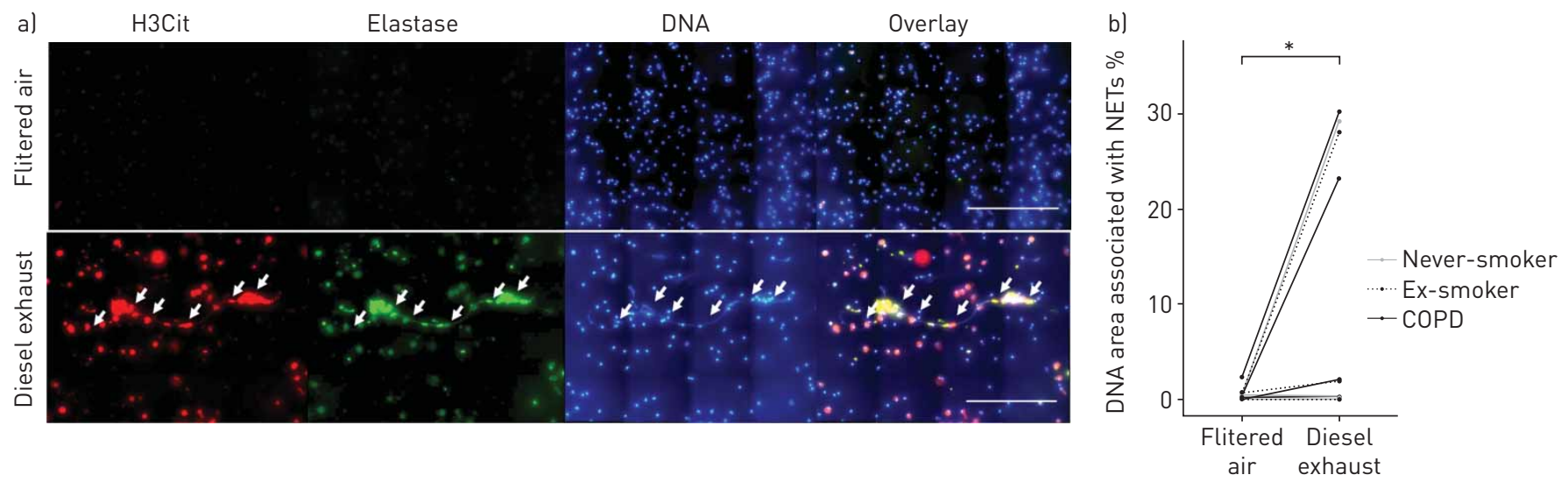

FIGURE 4 Representative neutrophil extracellular trap (NET) images in bronchoalveolar lavage (BAL). a) Representative images and quantification of NETs found in BAL following acute filtered air and diesel exhaust exposure $(n=15)$. Each panel shows a representative $4 \times 4$ stitched image of BAL after pelleting, resuspending and seeding onto glass slides, then staining for the following markers of NETs used to quantify BAL NETs: citrullinated histones (H3cit; red), neutrophil elastase (green) and DNA (blue (DAPI)). NETs can be identified as fibrous strands of elastase- and histone-coated DNA, visible $24 \mathrm{~h}$ following acute diesel exhaust exposure and denoted by white arrows. Normal intact cells following a filtered air exposure are shown for comparison. b) Images were quantified to assess the effect of diesel exhaust exposure on NET formation. The y-axis denotes slide area $\left(\mu \mathrm{m}^{2}\right)$ covered by NET markers divided by slide area $\left(\mu \mathrm{m}^{2}\right)$ covered by DNA $\times 100 \%$. Each line represents one participant. Scale bars $=250 \mu \mathrm{m} .{ }^{*}: \mathrm{p}<0.05$.

neutrophils as a key cell type mediating innate immune responses in the circulation and airways following

TRAP exposure. Indeed, previous studies have demonstrated that neutrophils migrate to the lungs following TRAP exposure $[12-15,28]$ and there is a significant body of evidence demonstrating that

a)

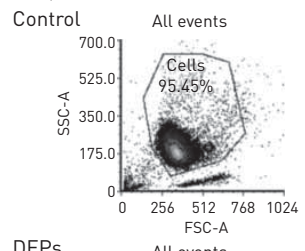

DEPs
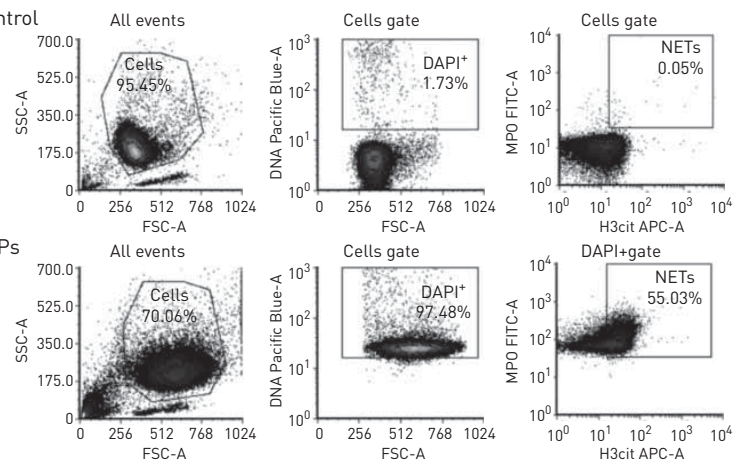

Cells gate
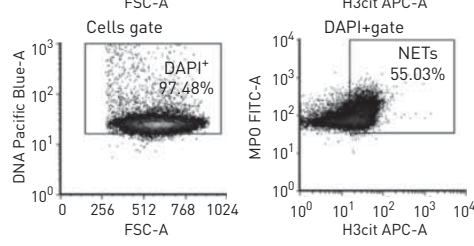

b)

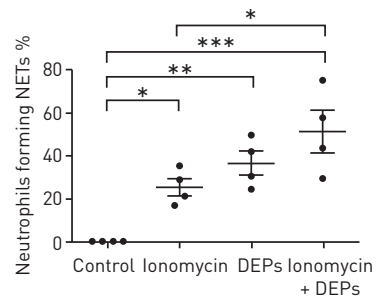

c)
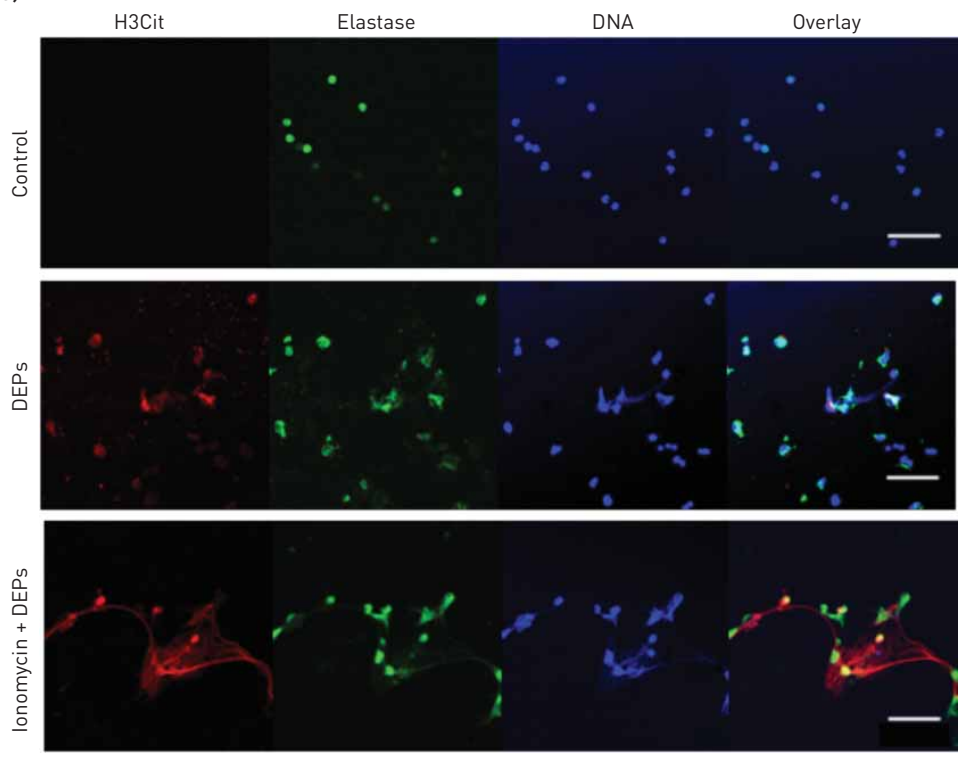

FIGURE 5 Diesel exhaust particles induce neutrophil extracellular trap (NET) formation in isolated peripheral blood neutrophils in vitro. a)

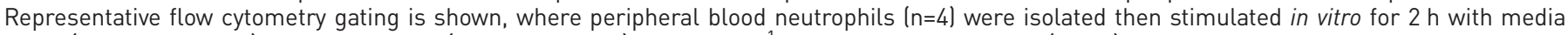
alone (negative control), $4 \mu \mathrm{M}$ ionomycin (positive control), $100 \mu \mathrm{g} \cdot \mathrm{mL}^{-1}$ diesel exhaust particles (DEPs) obtained from the outlet of our in-house

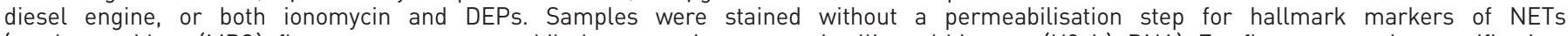
(myeloperoxidase (MPO), flow cytometry; neutrophil elastase, microscopy; citrullinated histones (H3cit), DNA). For flow cytometric quantification, cells were gated on based on forward scatter area (FSC-A) and side scatter area (SSC-A) to exclude debris and contaminating lymphocytes. Then $\mathrm{DAPI}^{+}$cells were gated on, followed by those cells expressing H3cit and MPO. NETs are defined as cells staining triple-positive for the aforementioned markers. b) lonomycin, DEPs and the combination significantly increased the percentage of neutrophils forming NETs, and adding DEPs to ionomycin significantly enhanced NET formation, when measured by flow cytometry. Data are presented as mean \pm SEM. $*$ : $p<0.05$, **: $p<0.01, * * *: p<0.001$. c) Representative immunocytochemistry images demonstrate formation of NETs after DEPs and ionomycin+DEPs, but not control. Scale bars $=50 \mu \mathrm{m}$. 
neutrophilic inflammation contributes to lung tissue damage [29], disease severity [17] and chronic inflammatory signalling [16].

Our data demonstrate that acute diesel exhaust exposure led to a more activated peripheral neutrophil phenotype (greater CD16, CXCR2 and activated CD11b expression) in participants with COPD compared to never-smokers. CD16 is a marker of neutrophil degranulation and activation [30], while CXCR2, the GRO- $\alpha$ and interleukin-8 receptor, is responsible for neutrophil trafficking to the lung [31] and is involved in some pathways of NET formation [32]. Evidence shows that CXCR2 plays an essential role in regulating NETosis in this population [32]. Therefore, elevated CXCR2 expression due to diesel exhaust exposure in those with COPD relative to never-smokers could increase neutrophil trafficking, NET formation and neutrophil responsiveness. Increased $\mathrm{CD} 11 \mathrm{~b}$ on neutrophil surfaces of COPD patients relative to never-smokers after diesel exhaust is also relevant, as this marker correlates with secreted proteases like matrix metalloproteinase (MMP)-8 and MMP-9 in a cigarette smoke extract model [33], both of which contribute to emphysematous tissue damage and correlate with severity of emphysema [34]. In addition, the activated form of this protein, which we demonstrate is increased in COPD patients exposed to diesel exhaust, makes up only a small portion of total CD11b on neutrophil surfaces, but is believed to be the most important functionally for its ability to interact with intercellular adhesion molecule-1 on activated endothelium, and is thus a key protein for neutrophil extravasation from the bloodstream [35]. These data show that peripheral neutrophils of COPD patients switch to a more activated state after diesel exhaust exposure than those of a never-smoker. Interestingly, there was no effect modification in ex-smokers, suggesting that this elevated risk was reserved to the COPD group in our acute exposure study. Although previous work postulates that ex-smokers have more pronounced lung inflammation even 1 year after quitting [36], it is possible that the degree of inflammatory signalling, or its persistent and relentless nature in COPD relative to ex-smokers, led COPD patients to a susceptibility threshold that was not met by ex-smokers in our study. In addition, COPD patients can experience greater pollutant deposition for the same level of exposure, increasing their effective exposure dose and thus susceptibility to inflammatory effects [37].

Band cells are a marker of bone marrow stimulation and release of neutrophils into the circulating pool from the bone marrow $[38,39]$. Observational studies show that peripheral band cells are increased by prolonged and high ambient air pollution and wood smoke exposure [38, 39], which contrasts with our results, and suggests that bone marrow stimulation may only occur above a certain exposure threshold. The reduction in circulating band cells after diesel exhaust shown herein is in agreement with previous work demonstrating a disappearance of band cells from the blood immediately following cigarette smoke exposure in an animal model [40], where band cell disappearance results from their limited deformability and sequestration in the lung microvasculature, contributing to lung tissue damage associated with smoke-induced emphysema [40,41]. This lends a conceivable mechanism through which band cells could contribute to lung tissue damage in a chronic TRAP exposure setting. However, without a tracer study we cannot confirm the reason for reduced band cell counts in the current study. The reduction in circulating neutrophils $24 \mathrm{~h}$ after exposure, with no increase in band cells, suggests that neutrophil recruitment to lung spaces seen in multiple previous acute diesel exhaust exposure studies [13-15, 42] may represent recruitment of existing neutrophil pools (and thus reduction in the percentage of peripheral neutrophils), rather than stimulation of neutrophil release from the bone marrow (in which case peripheral neutrophil levels would presumably remain normal or high, despite recruitment into lung spaces).

Acute diesel exhaust exposure increased BAL NET formation in 11 out of the 13 participants who had complete data. Increased amounts of NETs in the lung have been described in the context of COPD [20, 21], cigarette smoking [43] and e-cigarette use [44], but this is the first study to describe the same in an acute human TRAP exposure model. Clinically, increased NET formation is associated with COPD severity [22] and correlates with airflow limitation [21] in COPD patients. In addition, proteolytic neutrophil proteins contained in NETs are capable of damaging endothelium and epithelium [45, 46], while exposed histones can promote inflammation by acting as damage-associated molecular patterns [47]. Therefore, NET formation in the lung is a mechanism through which TRAP exposure could cause lung tissue damage characteristic of emphysema, and promote chronic inflammation in a repeated-exposure setting. Interestingly, four participants had particularly marked NET formation that was not explained by any particular patient phenotype, as there was at least one participant from each phenotype in this "high-responding" group, nor was it explained by smoking history or sex. Additional insight into which factors increase susceptibility to biological effects of TRAP exposure including NET formation, such as gene-environment interactions, may be useful.

Our in vitro findings were largely supportive of our in vivo results. Using flow cytometry and fluorescence microscopy, we showed that DEPs stimulate NET formation in peripheral neutrophils. In addition, the in vitro response to ionomycin was enhanced in the presence of DEPs, suggesting that ionomycin and DEPs 
may stimulate NETs through different pathways. Thus, DEPs deposited into the lung could be responsible for stimulating NET formation in neutrophils that travel to the site following exposure, with the caveat that blood neutrophils in this in vitro study may be phenotypically different from those in the lung [48]. Alternatively, some studies have demonstrated DEPs can translocate into the blood, where they would indeed interact with peripheral neutrophils, although probably at lower concentrations. In this case, the potential for NET formation in the vasculature has implications on known cardiovascular effects of TRAP exposure $[1,49,50]$.

One limitation of the current study was that BAL samples were used to assess neutrophil effects in the lung due to practical challenges of sample reliability (e.g. sputum) and low total cell counts (e.g. bronchial wash) in other samples. Neutrophil recruitment following diesel exhaust has been previously identified in bronchial wash and sputum [13-15, 42], and NETs have largely been studied in COPD sputum [20-22]. Nonetheless, a consistent NET response was still seen in presumably the more protected lung compartment (lower airways) for pollutant-induced neutrophilic inflammation. Another limitation is sample size: this study was powered to detect a main effect of diesel exhaust exposure, but we recognise the risk of Type II error associated with considering clinical phenotype as a modifying effect. Still, even with low numbers, we detected clear effect modification therein. Overall, this crossover study adds to the existing knowledge of neutrophil recruitment following diesel exhaust exposure, demonstrating that neutrophil activation is enhanced more in those with at-risk phenotypes. We postulate that acute diesel exhaust exposure results in recruitment of existing neutrophil pool, and show that diesel exhaust increases NET formation both in vitro and in vivo, highlighting novel mechanisms through which TRAP exposure may exert its harmful effects.

The Lancet Commission on Pollution and Health named research, including exploring emerging causal links between pollution, disease and subclinical impairment, as one of its six key recommendations to combat the massive global burden of pollution-related disease [1]. While a number of human exposure studies have begun to explain these mechanisms in healthy participants, much of the real-life burden occurs in vulnerable populations [1]. This study adds key mechanistic insight that can be used to support the policies pertaining to air pollution regulation that are paramount to public health protection, in addition to development or improvement of therapeutic approaches targeting neutrophilic inflammation in the context of air pollution exposure and respiratory disease.

Acknowledgements: We would like to extend our most sincere gratitude to the participants who volunteered their time to partake in this study. In addition, we thank the University of British Columbia, Vancouver General Hospital and Vancouver Coastal Health Research Institute for their support. The authors would like to thank Nafeez Syed and Jordan Guenette (University of British Columbia, Vancouver, BC, Canada) for assisting and providing facility for participant screening and $V_{\mathrm{O}_{2}}^{\prime} \max$ test, Wayne Tse (University of British Columbia) for his assistance in acquiring microscopy images, and Andrew Lee and Carley Schwartz (University of British Columbia) for collecting exposure data.

We would like to acknowledge grant funding from the Canadian Respiratory Research Network for this project. D.J. Wooding was supported by a Canada Graduate Scholarship (CIHR-CGSM), and a WorkSafeBC Training Award (RS2017-TG05). M.H. Ryu was supported by a Graduate Student Scholarship from Canadian Respiratory Research Network, WorkSafeBC Research Training Award (RS2016-TG08) and an NSERC Alexander Graham Bell Scholarship (CGS-D). H. Li was supported by International Program Fund for doctoral students from Sun Yat-sen University and the Chinese Government Scholarship. Funding information for this article has been deposited with the Crossref Funder Registry.

Conflict of interest: D.J. Wooding reports grants from WorkSafeBC and the Canadian Institutes of Health Research, during the conduct of the study. M.H. Ryu reports grants from Canadian Respiratory Research Network, WorkSafeBC and Natural Sciences and Engineering Research Council of Canada, during the conduct of the study. H. Li reports grants from Sun Yat-sen University International Program Fund and the Chinese Government (scholarship), during the conduct of the study. N.E. Alexis has nothing to disclose. O. Pena has nothing to disclose. C. Carlsten reports grants from Canadian Respiratory Research Network and the Canada Research Chairs Program, during the conduct of the study.

\section{References}

1 Landrigan PJ, Fuller R, Acosta NJ, et al. The Lancet Commission on pollution and health. Lancet 2018; 391 : $462-512$.

2 Lelieveld J, Evans J, Fnais M, et al. The contribution of outdoor air pollution sources to premature mortality on a global scale. Nature 2015; 525: 367-371.

3 Roth GA, Abate D, Abate KH, et al. Global, regional, and national age-sex-specific mortality for 282 causes of death in 195 countries and territories, 1980-2017: a systematic analysis for the Global Burden of Disease Study 2017. Lancet 2018; 392: 1736-1788.

4 Anderson H, Spix C, Medina S, et al. Air pollution and daily admissions for chronic obstructive pulmonary disease in 6 European cities: results from the APHEA project. Eur Respir J 1997; 10: 1064-1071.

5 Moolgavkar SH. Air pollution and hospital admissions for chronic obstructive pulmonary disease in three metropolitan areas in the United States. Inhal Toxicol 2000; 12: 75-90. 
6 Sunyer J, Sáez M, Murillo C, et al. Air pollution and emergency room admissions for chronic obstructive pulmonary disease: a 5-year study. Am J Epidemiol 1993; 137: 701-705.

7 Ko FW, Tam W, Wong TW, et al. Temporal relationship between air pollutants and hospital admissions for chronic obstructive pulmonary disease in Hong Kong. Thorax 2007; 62: 780-785.

8 Gan WQ, FitzGerald JM, Carlsten C, et al. Associations of ambient air pollution with chronic obstructive pulmonary disease hospitalization and mortality. Am J Respir Crit Care Med 2013; 187: 721-727.

9 Forbes LJ, Kapetanakis V, Rudnicka AR, et al. Chronic exposure to outdoor air pollution and lung function in adults. Thorax 2009; 64: 657-663.

10 Carlsten C, MacNutt MJ, Zhang Z, et al. Anti-oxidant N-acetylcysteine diminishes diesel exhaust-induced increased airway responsiveness in person with airway hyper-reactivity. Toxicol Sci 2014; 139: 479-487.

11 Carlsten C, Blomberg A, Pui M, et al. Diesel exhaust augments allergen-induced lower airway inflammation in allergic individuals: a controlled human exposure study. Thorax 2016; 71: 35-44.

12 Nordenhäll C, Pourazar J, Blomberg A, et al. Airway inflammation following exposure to diesel exhaust: a study of time kinetics using induced sputum. Eur Respir J 2000; 15: 1046-1051.

13 Salvi S, Blomberg A, Rudell B, et al. Acute inflammatory responses in the airways and peripheral blood after short-term exposure to diesel exhaust in healthy human volunteers. Am J Respir Crit Care Med 1999; 159: 702-709.

14 Behndig A, Mudway I, Brown J, et al. Airway antioxidant and inflammatory responses to diesel exhaust exposure in healthy humans. Eur Respir J 2006; 27: 359-365.

15 Stenfors N, Nordenhäll C, Salvi S, et al. Different airway inflammatory responses in asthmatic and healthy humans exposed to diesel. Eur Respir J 2004; 23: 82-86.

16 Mantovani A, Cassatella MA, Costantini C, et al. Neutrophils in the activation and regulation of innate and adaptive immunity. Nat Rev Immunol 2011; 11: 519-531.

17 Vlahos R, Wark PA, Anderson GP, et al. Glucocorticosteroids differentially regulate MMP-9 and neutrophil elastase in COPD. PLoS One 2012; 7: e33277.

18 Brinkmann V, Reichard U, Goosmann C, et al. Neutrophil extracellular traps kill bacteria. Science 2004; 303: $1532-1535$

19 Delgado-Rizo V, Martínez-Guzmán MA, Iñiguez-Gutierrez L, et al. Neutrophil extracellular traps and its implications in inflammation: an overview. Front Immunol 2017; 8: 81.

20 Pedersen F, Marwitz S, Holz O, et al. Neutrophil extracellular trap formation and extracellular DNA in sputum of stable COPD patients. Respir Med 2015; 109: 1360-1362.

21 Grabcanovic-Musija F, Obermayer A, Stoiber W, et al. Neutrophil extracellular trap (NET) formation characterises stable and exacerbated COPD and correlates with airflow limitation. Respir Res 2015; 16: 59.

22 Dicker AJ, Crichton ML, Pumphrey EG, et al. Neutrophil extracellular traps are associated with disease severity and microbiota diversity in patients with chronic obstructive pulmonary disease. J Allergy Clin Immunol 2018; 141: 117-127.

23 Birger N, Gould T, Stewart J, et al. The Air Pollution Exposure Laboratory (APEL) for controlled human exposure to diesel exhaust and other inhalants: characterization and comparison to existing facilities. Inhal Toxicol 2011; 23: 219-225.

24 Pronk A, Coble J, Stewart P. Occupational exposure to diesel engine exhaust: a literature review. J Expo Sci Environ Epidemiol 2009; 19: 443-457.

25 Freeman CM, Crudgington S, Stolberg VR, et al. Design of a multi-center immunophenotyping analysis of peripheral blood, sputum and bronchoalveolar lavage fluid in the Subpopulations and Intermediate Outcome Measures in COPD Study (SPIROMICS). J Transl Med 2015; 13: 19.

26 Gavillet M, Martinod K, Renella R, et al. Flow cytometric assay for direct quantification of neutrophil extracellular traps in blood samples. Am J Hematol 2015; 90: 1155-1158.

27 Naccache PH, Fernandes MJ. Challenges in the characterization of neutrophil extracellular traps: the truth is in the details. Eur J Immunol 2016; 46: 52-55.

28 Nightingale JA, Maggs R, Cullinan P, et al. Airway inflammation after controlled exposure to diesel exhaust particulates. Am J Respir Crit Care Med 2000; 162: 161-166.

29 Shapiro SD, Goldstein NM, Houghton AM, et al. Neutrophil elastase contributes to cigarette smoke-induced emphysema in mice. Am J Pathol 2003; 163: 2329-2335.

30 Faurschou M, Borregaard N. Neutrophil granules and secretory vesicles in inflammation. Microbes Infect 2003; 5: 1317-1327.

31 Virtala R, Ekman AK, Jansson L, et al. Airway inflammation evaluated in a human nasal lipopolysaccharide challenge model by investigating the effect of a CXCR2 inhibitor. Clin Exp Allergy 2012; 42: 590-596.

32 Pedersen F, Waschki B, Marwitz S, et al. Neutrophil extracellular trap formation is regulated by CXCR2 in COPD neutrophils. Eur Respir J 2018; 51: 1700970.

33 Friedrichs B, Neumann U, Schüller J, et al. Cigarette-smoke-induced priming of neutrophils from smokers and non-smokers for increased oxidative burst response is mediated by TNF- $\alpha$. Toxicol In Vitro 2014; 28 : 1249-1258.

34 Segura-Valdez L, Pardo A, Gaxiola M, et al. Upregulation of gelatinases A and B, collagenases 1 and 2, and increased parenchymal cell death in COPD. Chest 2000; 117: 684-694.

35 Diamond MS, Springer TA. A subpopulation of Mac-1 (CD11b/CD18) molecules mediates neutrophil adhesion to ICAM-1 and fibrinogen. I Cell Biol 1993; 120: 545-556.

36 Willemse BW, ten Hacken NH, Rutgers B, et al. Effect of 1-year smoking cessation on airway inflammation in COPD and asymptomatic smokers. Eur Respir J 2005; 26: 835-845.

37 Kim CS, Kang TC. Comparative measurement of lung deposition of inhaled fine particles in normal subjects and patients with obstructive airway disease. Am J Respir Crit Care Med 1997; 155: 899-905.

38 Sakai M, Sato Y, Sato S, et al. Effect of relocating to areas of reduced atmospheric particulate matter levels on the human circulating leukocyte count. J Appl Physiol 2004; 97: 1774-1780.

39 Swiston JR, Davidson W, Attridge S, et al. Wood smoke exposure induces a pulmonary and systemic inflammatory response in firefighters. Eur Respir J 2008; 32: 129-138.

40 Terashima T, Klut ME, English D, et al. Cigarette smoking causes sequestration of polymorphonuclear leukocytes released from the bone marrow in lung microvessels. Am J Respir Cell Mol Biol 1999; 20: 171-177. 
41 van Eeden SF, Kitagawa Y, Klut ME, et al. Polymorphonuclear leukocytes released from the bone marrow preferentially sequester in lung microvessels. Microcirculation 1997; 4: 369-380.

42 Salvi SS, Nordenhall C, Blomberg A, et al. Acute exposure to diesel exhaust increases IL-8 and GRO- $\alpha$ production in healthy human airways. Am J Respir Crit Care Med 2000; 161: 550-557.

43 Qiu S-L, Zhang H, Tang QY, et al. Neutrophil extracellular traps induced by cigarette smoke activate plasmacytoid dendritic cells. Thorax 2017; 72: 1084-1093.

44 Reidel B, Radicioni G, Clapp PW, et al. E-cigarette use causes a unique innate immune response in the lung, involving increased neutrophilic activation and altered mucin secretion. Am J Respir Crit Care Med 2018; 197: 492-501.

45 Fuchs TA, Abed U, Goosmann C, et al. Novel cell death program leads to neutrophil extracellular traps. J Cell Biol 2007; 176: 231-241.

46 Saffarzadeh M, Juenemann C, Queisser MA, et al. Neutrophil extracellular traps directly induce epithelial and endothelial cell death: a predominant role of histones. PLoS One 2012; 7: e32366.

47 Allam R, Kumar SV, Darisipudi MN, et al. Extracellular histones in tissue injury and inflammation. $J$ Mol Med 2014; 92: 465-472.

48 Fortunati E, Kazemier K, Grutters J, et al. Human neutrophils switch to an activated phenotype after homing to the lung irrespective of inflammatory disease. Clin Exp Immunol 2009; 155: 559-566.

49 Mills NL, Törnqvist H, Robinson SD, et al. Diesel exhaust inhalation causes vascular dysfunction and impaired endogenous fibrinolysis. Circulation 2005; 112: 3930-3936.

50 Törnqvist H, Mills NL, Gonzalez M, et al. Persistent endothelial dysfunction in humans after diesel exhaust inhalation. Am J Respir Crit Care Med 2007; 176: 395-400. 